\title{
Quo vadis with the $Q$ tracts
}

Glutamine Repeats and Neurodegenerative Diseases: Molecular Aspects

edited by Peter S. Harper and Max Perutz

Oxford University Press · January 2001

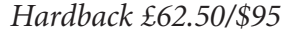

F. Ulrich Hartl and Peter Breuer

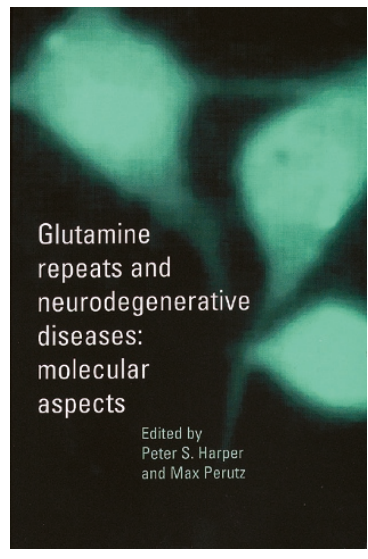

$\mathrm{R}$

esearch on polyglutamine diseases is heralding its second decade. Many advances have been made in this fastmoving field since the first identification of an expanded polyglutamine tract in the androgen receptor as the cause of Kennedy's disease. Since then, several other neurodegenerative disorders, including Huntington's disease and the various ataxias, have been found to be caused by the elongation of polyQ sequences. These polyQ expansions occur in otherwise unrelated proteins and result in neurotoxicity by a common pathogenetic process.

Soon after the isolation and identification of the Huntington's Disease (HD) gene in 1993 it was Max Perutz who provided seminal insight into the molecular basis of polyQ diseases by realizing the potential of $\beta$-strands of poly-L-glutamine to self-associate into so-called polar zippers. Critical experimental support for the polar zipper hypothesis followed with the discovery by Gillian Bates and co-workers that mice expressing a fragment of the HD gene containing the expanded polyQ sequence develop intranuclear aggregates in the brain. Erich Wanker and colleagues then demonstrated that this aggregation process is faithfully reproduced in vitro with recombinant polyQ proteins.

It is now generally accepted that a unifying feature of pathologically elongated polyQ tracts is to cause the aggregation of the proteins in which they occur. A new book on the molecular aspects of polyQ diseases edited by Peter Harper and Max Perutz deals with the fascinating questions raised by these insights, including one that is most intensely debated by experts and interested observers: are the polyQ aggregates the direct cause of neurodegeneration, or are they merely an epiphenomenon of a disease process to which polyQ proteins contribute by a so far unknown mechanism?

Glutamine Repeats and Neurodegenerative Diseases: Molecular Aspects is a compilation of updated contributions by leading scientists originally submitted on the occasion of a Royal Society discussion meeting in 1998. After an excellent, clinically oriented introduction by Harper on $\mathrm{HD}$, the most prominent polyQ disorder, it starts off with a review of various animal models now available for the study of this disease. Although most of these models utilize transgenic mice, the reader also learns about the potential of the Drosophila system in analysing polyQ-induced neurotoxicity. Indeed, a recent search for suppressors of polyQ-mediated neurotoxicity in transgenic flies revealed the remarkable capacity of certain molecular chaperones to protect neurons against degeneration and death.

Other chapters deal with the molecular basis of polyQ toxicity, the biochemistry of polyQ proteins and the genetic mechanisms underlying the pathological expansion of the CAG trinucleotide repeats encoding polyQ sequences. The last part of the book describes how research on HD and other polyQ diseases promises to unify current understanding of the molecular pathology forming the basis of a larger group of neurodegenerative diseases. Alzheimer's and Parkinson's disease as well as the prion diseases are all associated with the deposition of amyloid-like protein aggregates in neurons and these deposits have characteristic features in common with the aggregates found in HD.

As stated in the preface, the intention of this book is to stimulate further a field that already is very active, and to make it more accessible to clinicians and workers in allied areas of neuroscience. This goal is successfully achieved both by the breadth of coverage and the quality of the individual contributions. Hopefully many readers will be inspired to explore further the intriguing ways of the polyQ tracts.

F. Ulrich Hartl and Peter Breuer are in the Max-Planck-Institute of Biochemistry, Am Klopferspitz 18A, D-82152 Martinsried, Germany.

e-mailuhartl@biochem.mpg.de

\section{Other Neurodegeneration Books}

Advances in Research on Neurodegeneration: Etiopathogenesis edited by Y. Mizuno, D. B. Caine and R. Horowski

Birkhauser, £90.85/\$104.50

Neurodegeneration and

Neuroprotection in Parkinson's Disease edited by C. W. Olanow, P. Jenner and M. Youdin

Springer, $£ 76 / \$ 80$

Neuroprotection in Neurodegeneration edited by P. Riederer, J. Fritze and M.

Youdin

Springer, $£ 56.50 / \$ 73$

Neurodegeneration Methods and Protocols

edited by Jean Harry and Hugh A. Tilson Humana, £72.50/\$89.50

Advances in Research on Neurodegeneration Volume 8 edited by P. Riederer, D. Calne, R. Horowski and Y. Mazuno Springer, £85.50/\$124

\section{Other Huntington's Disease Books}

Huntington's Disease

by Oliver Quarrell

Oxford University Press, $\mathfrak{£ 1 1 . 9 9 / \$ 1 9 . 9 5}$

Devil's Dance

by Richard R. Karlen

Ironbound Press, $£ 10.60 / \$ 15$

Living with Huntington's Disease by Dennis Phillips

University of Wisconsin Press, $£ 25.50$

Huntington's Disease by Peter Harper W.B. Saunders, $£ 59$

Neural Transplantation for

Huntington's Disease

by P. R. Sanberg

CRC, $£ 85$ 